\title{
RIGIDITY AND OTHER TOPOLOGICAL ASPECTS OF COMPACT NONPOSITIVELY CURVED MANIFOLDS
}

\author{
F. T. FARRELL AND L. E. JONES
}

\begin{abstract}
Let $M$ be a compact connected Riemannian manifold whose sectional curvature values are all nonpositive. Let $\Gamma$ denote the fundamental group of $M$. We prove that any homotopy equivalence $f: N \rightarrow M$ from a compact closed manifold $N$ is homotopic to a homeomorphism, provided that $m \geq 5$ where $m=\operatorname{dim} M$. We show that the surgery $L$-group $L_{k+m}\left(\Gamma, w_{1}\right)$ is isomorphic to the set of homotopy classes of maps $\left[M \times I^{k}\right.$ rel $\left.\partial, G / \mathrm{TOP}\right]$, where $I^{k}$ is the $k$-dimensional cube (with $k>0$ ). We also show that the Whitehead group $\mathrm{Wh}(\Gamma)$, the projective class group $\widetilde{K}_{0}(Z \Gamma)$, and the lower $K$ groups $K_{-n}(Z \Gamma), n \geq 1$, are all isomorphic to the one element group. The higher $K$-groups $K_{n}(Z \Gamma), n \geq 0$, are computed up to rational isomorphism type. All of these results have previously been obtained by the authors in the case that the sectional curvature values of $M$ are strictly negative (cf. $[7,8,9,10])$.
\end{abstract}

In all the following results we let $M$ denote a compact connected Riemannian manifold all of whose sectional curvature values are nonpositive, and we let $\Gamma$ denote the fundamental group of $M$.

Theorem 1. If $h: N \rightarrow M$ is a homotopy equivalence from a compact closed manifold $N$, and if $\operatorname{dim}(M) \geq 5$, then there is a homotopy of $h$ to a homeomorphism.

Let $\mathscr{P}(M)$ denote the semisimplicial space of stable topological pseudo-isotopies of $M$. For any stratified fibration $p: E \rightarrow B$ we let $\mathscr{P}(E ; p)$ denote the semisimplicial space of compactly supported stable topological pseudo-isotopies on $E$ which have arbitrarily small control in $B$ (defined in [23]). If $f: E \rightarrow M$ is a continuous map then denote by $F: \mathscr{P}(E ; p) \rightarrow \mathscr{P}(M)$ the map which is induced by $f$.

Received by the editors April 3, 1989 and, in revised form, June 27, 1989.

1980 Mathematics Subject Classification (1985 Revision). Primary 18F25, 22E40, 57D50.

Both authors were supported in part by the NSF.

(C) 1990 American Mathematical Society $0273-0979 / 90 \$ 1.00+\$ .25$ per page 
Theorem 2. There is a stratified fibration $p: E \rightarrow B$, each fiber of which is a circle, and a continuous map $f: E \rightarrow M$. The induced map $F: \mathscr{P}(E ; p) \rightarrow \mathscr{P}(M)$ is a homotopy equivalence.

Let $\mathscr{P}_{*}\left(S^{1}\right)$ denote the $\Omega$-spectrum defined as follows: $\mathscr{P}_{0}\left(S^{1}\right)$ $=\mathscr{P}\left(S^{1}\right) ; \mathscr{P}_{i}\left(S^{1}\right)$ is the $i$ th loop space of $\mathscr{P}\left(S^{1}\right)$ for any $i<0$; $\mathscr{P}_{i}\left(S^{1}\right)$ is the standard $i$ th delooping of $\mathscr{P}\left(S^{1}\right)$ for any $i>$ 0 (cf. [17, Appendix II]). We remark that $\mathscr{P}(E ; p)$ can be effectively computed from $\mathscr{P}_{*}\left(S^{1}\right)$ and $p: E \rightarrow B$; for example $\pi_{k}(\mathscr{P}(E ; p))$ is equal to the $k$ th homology group of $B$ with "stratified and twisted coefficients" $\mathscr{P}_{*}\left(S^{1}\right)$, where the stratification and twisting of the coefficients $\mathscr{P}_{*}\left(S^{1}\right)$ is induced by that of the fibration $p: E \rightarrow B$ (cf. [23, Appendix]). This points out the importance of understanding the fibration $p: E \rightarrow B$ of Theorem 2.

If $M$ has strictly negative sectional curvature values then $B$ of Theorem 2 is a countable infinite discrete space. (This version of Theorem 2 was proven by the authors in [8].) In general the $p: E \rightarrow B$ of Theorem 2 is obtained as follows. Let $S(M)$ and $R P(M)$ denote the unit sphere bundle of $M$ and the real projective bundle for $M$. The orbits of the geodesic flow on $S(M)$ cover the leaves of a one-dimensional foliation $\mathscr{G}$ for $R P(M)$. For each $t>0$ let $E_{t}$ denote the union of all the closed leaves of $\mathscr{G}$ which have length less than or equal to $t$, and let $p_{t}: E_{t} \rightarrow B_{t}$ denote the quotient map obtained by collapsing each closed leaf in $E_{t}$ to a point. Finally let $p: E \rightarrow B$ denote the direct limit as $t \rightarrow \infty$ of the maps $p_{t}: E_{t} \rightarrow B_{t}$. Let $f: E \rightarrow M$ denote the direct limit of the composite maps $E_{t} \stackrel{\text { incl }}{\longrightarrow} R P(M) \stackrel{\text { proj }}{\longrightarrow} M$.

\section{Calculation of the $L$-GROUPS}

Let $I^{k}$ denote the $k$-dimensional cube. There is a more general version of Theorem 1 which states that any homotopy equivalence $h:(N, \partial N) \rightarrow\left(M \times I^{k}, M \times \partial I^{k}\right)$ from the compact manifold pair $(N, \partial N)$-such that $h: \partial N \rightarrow M \times \partial I^{k}$ is already a homeomorphism-can be homotoped to a homeomorphism modulo $h \mid \partial N$, provided that $\operatorname{dim}(M)+k \geq 5$. This more general version of Theorem 1 has as a consequence that the surgery homomorphism

$$
\theta:\left[M \times I^{k} \operatorname{rel} \partial, G / \mathrm{TOP}\right] \rightarrow L_{m+k}\left(\Gamma, w_{1}\right)
$$


is an isomorphism, provided that $m+k>5$ and $k>0$. (Here $w_{1}: \Gamma \rightarrow Z_{2}$ is the homomorphism determined by the first StiefelWhitney class of $M, m=\operatorname{dim}(M)$, and $L_{m+k}\left(\Gamma, w_{1}\right)$ is the $(m+k)$-dimensional surgery group for $\Gamma$ with orientation data $w_{1}$ which is defined in [26].)

\section{Calculation of the $K$-groups and Whitehead Groups}

Let $R^{k}$ denote $k$-dimensional Euclidean space. There is the following more general version of Theorem 2 . Let $\mathscr{P}_{b}\left(M \times R^{k}\right)$ denote the semisimplicial space of all stable topological pseudoisotopies on $M \times R^{k}$ which are bounded in the $R^{k}$-factor. Let $\mathscr{P}_{b}^{k}(E ; p)$ denote the semisimplicial space of all stable topological pseudo-isotopies on $E \times R^{k}$ which are bounded in the $R^{k}$-factor and which have arbitrary small control in $B$ (with respect to the composite projection $E \times R^{k} \stackrel{\text { proj }}{\longrightarrow} E \stackrel{p}{\longrightarrow} B$ ). Then $f: E \rightarrow M$ induces a homotopy equivalence $F_{k}: \mathscr{P}_{b}^{k}(E ; p) \rightarrow \mathscr{P}_{b}\left(M \times R^{k}\right)$. This more general version of Theorem 2 has the following consequences (which can be deduced from it as in [8 and 9, Appendix]): $\operatorname{Wh}_{n}(\Gamma) \otimes Z(1 / N)=0$, for any $n \geq 1$ and $N=$ $[(n+1) / 2] ! ; K_{n}(Z \Gamma)=0$ for any $n<0 ; \widetilde{K}_{0}(Z \Gamma)=0 ; K_{n}(Z \Gamma) \otimes$ $Q=H_{n}(M, Q) \oplus\left(\bigoplus_{i \geq 1} H_{n-1-4 i}(M, Q)\right)$ for any $n$.

THE SPACE OF SELF HOMEOMORPHISMS OF $M$

Let $H(M)$ denote the space of self homeomorphisms of $M$, and let $g: H(M) \rightarrow \operatorname{Out}(\Gamma)$ denote the forgetful map to the outerautomorphism group of $\Gamma$. Note that Theorem 1 implies that $g$ is onto. Let $H_{0}(M)$ denote the kernel of $g$. Then if $m>10$ (where $m=\operatorname{dim}(M))$, and if $n, N$ are integers with $0 \leq n \leq(m-7) / 3$ and $N=[(n+4) / 2]$ !, we have that

$$
\pi_{n}\left(H_{0}(M)\right) \otimes Z(1 / N)= \begin{cases}0, & \text { if } n \neq 1 \\ \operatorname{center}(\Gamma) \otimes Z\left(\frac{1}{2}\right), & \text { if } n=1 .\end{cases}
$$

Remark. The reader is referred to the following references: to [6, $9,10,15]$ for results related to Theorem $1 ;$ to $[8,21,25]$ for results related to Theorem 2 ; to $[5,9,10,15]$ for results related to the $L$-group calculations; to $[4,7,8,14,18,21,24]$ for results related to the $K$-group and Whitehead group calculations. To obtain their results the author used in an important way results from the following references: $[3,5,17,19,20,21,22,23,25,26]$.

Proofs of Theorems 1 and 2. The proofs for Theorems 1 and 2 are similar in spirit to the proofs of these same theorems given in $[7,8$, $9,10]$ for the special case when $M$ has strictly negative sectional 
curvature values. However, there are several important technical differences, one of which we will discuss now in the context of the proof for Theorem 1.

To prove Theorem 1 for $M$ of strictly negative sectional curvature the authors use in $[9,10]$ a foliated control theorem for structure sets, where the foliations have the following important properties:

(a) The leaves have dimension one.

(b) For any $\alpha>0$ there are only finitely many leaves which have length less than $\alpha$.

This type of foliated control theorem unfortunately does not apply to the relevant foliations when $M$ is allowed to have some zero sectional curvature values. (For example there are whole continuous families of closed orbits of bounded length for the geodesic flow on the unit sphere bundle of the torus $T^{n}$. So this foliation doesn't satisfy (b) above.) We have therefore had to extend the foliated control theory for structure sets used in $[9,10]$. The following extension is proven in [12].

Let $N$ denote a compact closed smooth manifold and let $\mathscr{F}$ denote a $C^{2}$ foliation of $N$ by one-dimensional leaves. Let $p: E \rightarrow$ $N$ denote a fiber bundle over $N$ which has a compact closed manifold for fiber. Let $h: X \rightarrow E$ denote a homotopy equivalence from the compact closed manifold $X$. We refer the reader to [12, $13]$ for the meaning of $h$ being $(\alpha, \varepsilon)$-controlled over $(N, \mathscr{F})$ for numbers $\alpha, \varepsilon>0$. See [12, Appendix] for a proof of the following theorem.

Theorem 3. There is an integer $k>0$ which depends only on $\operatorname{dim}(N)$. Given any $\alpha>0$ there is $\varepsilon>0$. If the homotopy equivalence $h: X \rightarrow E$ is $(\alpha, \varepsilon)$-controlled over $(N, \mathscr{F})$ then there is a homotopy $H_{t}: X \times T^{k} \rightarrow E \times T^{k}, t \in[0,1]$, of $h \times 1: X \times T^{k} \rightarrow E \times T^{k}$ to a map $H_{1}$ which is split over a triangulation of $N$ (here 1: $T^{k} \rightarrow T^{k}$ is the identity map on the $k$-dimensional torus).

\section{REFERENCES}

1. W. Ballmann, M. Gromov, and V. Schroeder, Manifolds of non-positive curvature, Birkhäuser, Boston, 1985.

2. H. Bass, A. Heller, and R. G. Swan, The Whitehead group of a polynomial extension, Inst. Hautes Études Sci. Publ. Math. 22 (1964), 61-79.

3. D. Burghelea and R. Lashof, Stability of concordances and the suspension homomorphism, Ann. of Math. (2) 105 (1977), 449-472. 
4. F. T. Farrell and W.-C. Hsiang, The Whitehead group of poly- (finite or cyclic) groups, J. London Math. Soc. 24 (1981), 308-324.

5. _ On Novikov's conjecture for nonpositively curved manifolds. I, Ann. of Math. (2) 113 (1981), 199-209.

6. _ Topological characterizations of flat and almost flat Riemannian manifolds $M^{n}(n \neq 3,4)$, Amer. J. Math. 105 (1983), 641-672.

7. F. T. Farrell and L. E. Jones, $K$-theory and dynamics. I, Ann. of Math. (2) 124 (1986), 531-569.

8. __ K-theory and dynamics. II, Ann. of Math. (2) 126 (1987), 451-493.

9. __ A topological analogue of Mostow's rigidity theorem, J. Amer. Math. Soc. 2 (1989), 257-370.

10. _ Compact negatively curved manifolds (of $\operatorname{dim} \neq 3,4$ ) are topological rigid, Proc. Nat. Acad. Sci. 86 (1989), 3461-3463.

11. - Negatively curved manifolds with exotic smooth structures, J. Amer. Math. Soc. 2 (1989), 899-908.

12. _ Foliated control without radius of injectivity restrictions, Topology (in press).

13. $\ldots$, Foliated control theory. I and II, K-theory 2 (1988), 357-399 and 401430.

14. _ Algebraic K-theory of discrete subgroups of Lie groups, Proc. Nat. Acad. Sci. 84 (1987), 3095-3096.

15. _ _ The surgery L-groups of poly-(finite or cyclic) groups, Invent. Math. 91 (1988), 559-586.

16. D. Gromoll and J. Wolf, Some relations between the metric structure and the algebraic structure of the fundamental group in manifolds of non-positive curvature, Bull. Amer. Math. Soc. 77 (1971), 545-552.

17. A. Hatcher, Concordance spaces, higher simple homotopy theory, and applications, Proc. Sympos. Pure Math. 32 (1978), 3-21.

18. B. Hu, Ph. D. Thesis, SUNY at Stony Brook, 1989.

19. K. Igusa, The stability theorem for smooth pseudo-isotopies, $K$-Theory 2 (1988), 1-355.

20. R. C. Kirby and L. C. Siebenmann, Foundational essays on topological manifolds, smoothings, and triangulations, Ann. of Math. Studies 88, Princeton Univ. Press, Princeton, N.J., 1977.

21. A. J. Nicas, On the higher Whitehead groups of a Bieberbach group, Trans. Amer. Math. Soc. 287 (1985), 853-859.

22. F. Quinn, Ends of maps. I, Ann. of Math. (2) 110 (1979), 275-331.

23. __ Ends of maps. II, Invent. Math. 68 (1982), 353-424.

24. _ Algebraic K-theory of poly-(finite or cyclic) groups, Bull. Amer. Math. Soc. (N.S.) 12 (1985), 221-226.

25. F. Waldhausen, Algebraic $K$-theory of topological spaces I, Proc. Sympos. Pure Math., vol. 32, Amer. Math. Soc., Providence, R.I., 1978, pp. 35-60.

26. C. T. C. Wall, Surgery on compact manifolds, Academic Press, New York, 1970.

27. S.-T. Yau, Seminar on differential geometry, Annals. of Math. Studies, Princeton Univ. Press, Princeton, N.J., 1982. 
28. __ On the fundamental group of compact manifolds of non-positive curvature, Ann. of Math. (2) 93 (1971), 579-585.

Department of Mathematics, Columbia University, New York, New YORK 10027

Department of Mathematics, State University of New York, Stony BROOK, NEW YORK 11794 